\title{
Agradecimentos aos revisores 2020
}

\section{Acknowledgement to Reviewers 2020}

Os altos padrões científicos mantidos pela Revista Portuguesa de Investigação Comportamental e Social nos seus artigos deveram muito ao esforço dos revisores, que ofereceram livremente o seu tempo e conhecimento. Assim, os Editores da Revista Portuguesa de Investigação Comportamental e Social agradecem aos seguintes revisores que reviram artigos no período de novembro de 2019 a novembro de 2020.

The high scientific standards maintained by the Portuguese Journal of Behavioral and Social Research in its papers owe much to the effort reviewers, who gave their time freely, and knowledge. Thus, the Editors of the Portuguese Journal of Behavioral and Social Research acknowledge with particular gratitude the following reviewers who have reviewed papers during the period November 2019-November 2020.

Ana Gomes, Prof.

Universidade Autónoma de Lisboa, Portugal

Anna Laura Albuquerque, Mestre

Dialética Social Qualificação e Aperfeiçoamento profissional, Portugal

Carla Cerqueira, Prof.

Universidade Lusófona do Porto, Portugal

Everton Garcia da Costa, Prof.

Instituto Federal Sul-Rio-Grandense, Brasil

Fátima Regina Ney Matos, Prof.

Instituto Superior Miguel Torga, Portugal

Fernanda Bento Daniel, Prof. (2x)

Instituto Superior Miguel Torga, Portugal

Francisco Sampaio, Prof.

Fundação Ensino e Cultura Fernando Pessoa, Portugal

\section{DI\&D | ISMT}

rpics@ismt.pt

https://rpics.ismt.pt 
Helena Grangeia, Prof.

Instituto Universitário da Maia, Portugal

Helena Loureiro, Prof.

Universidade de Aveiro, Portugal

Isabel Silva, Prof.

Universidade Fernando Pessoa, Portugal

Joselany Áfio Caetano, Prof.

Universidade Federal do Ceará, Brasil

Juliana Pedro, Prof

Centro de Psicologia da Universidade do Porto, Portugal

Madalena Sofia Oliveira, Prof.

Instituto Universitário de Ciências da Saúde. Cooperativa de Ensino Superior Politécnico e Universitário, Portugal

Maria José Nogueira, Prof.

Escola Superior de Enfermagem da Cruz Vermelha Portuguesa - Alto Tâmega, Portugal

Mariana Vaz Pires Marques, Prof.

Centro de Responsabilidade Integrado de Psiquiatria do Centro Hospitalar e Universitário de Coimbra, Portugal

Marina Rezende Bazon, Prof.

Universidade de São Paulo, Brasil

Marlene Braz Rodrigues, Prof.

Universidade Lusófona de Humanidades e Tecnologias, Portugal

Rui Manuel Sousa Mendes, Prof.

Instituto Politécnico de Coimbra. Escola Superior de Educação de Coimbra, Portugal

Rui Oliveira Moreira, Dr.

Instituto Estadual de Diabetes e Endocrinologia.

Sociedade Brasileira de Endocrinologia e Metabologia, Brasil

Sofia Balula Dias, Prof.

Faculdade de Motricidade Humana, Universidade de Lisboa, Portugal

Sónia Catarina Carvalho Simões Prof.

Instituto Superior Miguel Torga, Portugal

Susana Isabel Vicente Ramos, Prof.

Universidade de Coimbra, Centro de Investigação do Desporto e da Actividade Física, Portugal

Teresa Medeiros, Prof.

Universidade dos Açores, Ponta Delgada; Instituto de Psicologia Cognitiva, Desenvolvimento Humano e Social, Coimbra,

Portugal 